April 19, 2010; ACCePted to the Astronomical Journal

Preprint typeset using $\mathrm{LTTEX}_{\mathrm{E}}$ style emulateapj v. 11/10/09

\title{
THE NATURE OF THE STRONG 24 MICRON SPITZER SOURCE J222557+601148: NOT A YOUNG GALACTIC SUPERNOVA REMNANT
}

\author{
Robert A. Fesen \& Dan Milisavljevic \\ 6127 Wilder Lab, Department of Physics \& Astronomy, Dartmouth College, Hanover, NH 03755 \\ April 19, 2010; Accepted to the Astronomical Journal
}

\begin{abstract}
The nebula J222557+601148, tentatively identified by Morris et al. (2006) as a young Galactic supernova remnant (SNR) from Spitzer Galactic First Look Survey images and a follow-up midinfrared spectrum, is unlikely to be a SNR remnant based on $\mathrm{H} \alpha$, [O III], [S II] images and low dispersion optical spectra. The object is seen in $\mathrm{H} \alpha$ and [O III] $\lambda 5007$ images as a faint, roughly circular ring nebula with dimensions matching that seen in $24 \mu \mathrm{m}$ Spitzer images. Low-dispersion optical spectra show it to have narrow $\mathrm{H} \alpha$ and [N II] $\lambda \lambda 6548,6583$ line emissions with no evidence of broad or high-velocity $\left(\mathrm{v} \geq 300 \mathrm{~km} \mathrm{~s}^{-1}\right)$ line emissions. The absence of any high-velocity optical features, the presence of relatively strong [N II] emissions, a lack of detected [S II] emission which would indicate the presence of shock-heated gas, plus no coincident X-ray or nonthermal radio emissions indicate the nebula is unlikely to be a SNR, young or old. Instead, it is likely a faint, high-excitation planetary nebula $(\mathrm{PN})$ as its elliptical morphology would suggest, lying at a distance $\sim 2-3 \mathrm{kpc}$ with unusual but not extraordinary mid-IR colors and spectrum. We have identified a $m_{r^{\prime}}=22.4 \pm 0.2$ star as a PN central star candidate.

Subject headings: ISM: supernova remnant - planetary nebulae: individual (J222557+601148)
\end{abstract}

\section{INTRODUCTION}

Of the 274 Galactic supernova remnants (SNRs) currently known (Green 2009), few are believed to be relatively young with ages of 3000 years or less. The list of young Galactic SNRs includes the historic guest stars of SN 1604 (Kepler's SNR), SN 1572 (Tycho's SNR), SN 1054 (Crab Nebula), SN 1006 (G327.6+14.6), SN 386 (G11.2-0.3), and SN 185 (RCW 86).

There are also about a dozen remnants which do not have firmly established ages but are probably less than a few thousand years old. These include the recently recognized very young remnant G1.9+0.3 (age $\simeq 150 \mathrm{yr}$; Reynolds et al. 2008, Green et al. 2008) and Cassiopeia $\mathrm{A} \quad(\mathrm{SN} \approx 1680$; Thorstensen et al. 2001, Fesen et al. 2006)

With so few young SNRs known, the discovery of even one more young Galactic SNR is significant. Young SNRs are of special interest since they offer a host of details on supernova explosions on finer spatial scales than from extragalactic SNR investigations, including expansion asymmetries, ejecta abundances, and clues regarding the nature of the progenitor star and its pre-SN environment.

Hence the discovery of a small symmetric nebula, SSTGFLS J222557+601148 (hereafter J222557), detected in $24 \mu \mathrm{m}$ Spitzer Galactic First Look Survey (GFLS) images and tentatively identified as a possible SNR with an age $\sim 1000$ yr by Morris et al. (2006) is worth investigating. Here we present optical images and spectra which indicate this nebula is likely not a supernova remnant but instead a faint, high-excitation planetary nebula $(\mathrm{PN})$.

\section{OBSERVATIONS}

Both narrow and broad passband optical images of J222557 were obtained in September 2006 using a backside illuminated 2048 × 2048 SITe CCD detector attached to the McGraw-Hill $1.3 \mathrm{~m}$ telescope at the MDM Obser- vatory (MDM) at Kitt Peak. The CCD's 24 micron size pixels gave an image scale of 0.508 and a field of view of approximately $17^{\prime}$ square.

The nebula was imaged on 2006 Sept 27 using a pair of matched on and off $\mathrm{H} \alpha$ interference filters centered at 6568 and $6510 \AA$ (FWHM $=30 \AA)$, a [O III] $\lambda 5007$ filter $(\mathrm{FWHM}=30 \AA$ ), and a $[\mathrm{S} \mathrm{II}] \lambda \lambda 6716,6731$ filter (FWHM $=50 \AA$ ). On Sept 28 broadband images using a Harris B filter were also taken. Individual image exposure times for all filters were $1000 \mathrm{~s}$ taken in sets of two or three.

Additional images of J222557 were obtained on 2008 July 30 using the MDM $2.4 \mathrm{~m}$ Hiltner telescope with the RETROCAM CCD camera (Morgan et al. 2005). Images were taken using SDSS filters $g^{\prime}$ and $r^{\prime}$ with exposure times of $2 \times 1200$ and $2 \times 900 \mathrm{~s}$. Conditions at the time of the observations were believed to be photometric with seeing around $1.5^{\prime \prime}$. Images were flux calibrated with standard stars from Smith et al. (2002). Standard pipeline data reduction of all images was performed using IRAF/STSDASI. This included debiasing, flat-fielding, and cosmic ray and hot pixel removal.

Follow-up low-dispersion optical spectra of J222557 were obtained on 2008 July 31 using the MDM $2.4 \mathrm{~m}$ telescope with a Modular Spectrograph and $2048 \times 2048$ pixel SITe CCD detector. A north-south $1.2^{\prime \prime} \times 5^{\prime}$ slit and a 600 line $\mathrm{mm}^{-1} 5000 \AA$ blaze grism was used to obtain $2 \times 900 \mathrm{~s}$ exposures spanning the spectral region $4300-7500 \AA$ with a resolution of $6 \AA$. The slit was placed over the bright southeast region as seen in the 24 $\mu \mathrm{m}$ Spitzer image. Conditions were good but not photo-

\footnotetext{
1 IRAF is distributed by the National Optical Astronomy Observatories, which is operated by the Association of Universities for Research in Astronomy, Inc. (AURA) under cooperative agreement with the National Science Foundation. The Space Telescope Science Data Analysis System (STSDAS) is distributed by the Space Telescope Science Institute.
} 


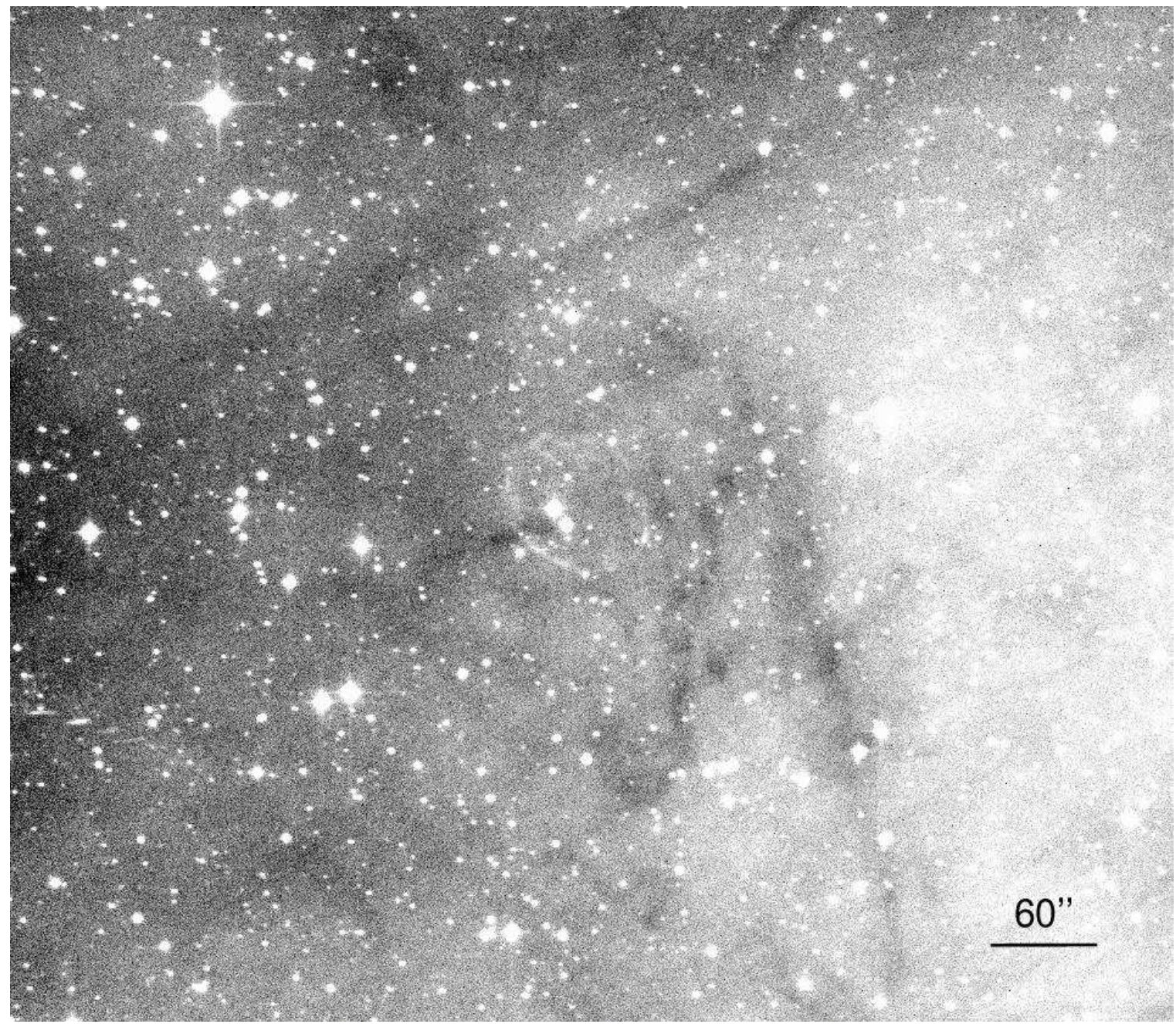

FIG. 1. - H $\alpha$ image of the J222557+601148 region showing the detection of a faint emission shell. North is up and East to the left.

metric with $1.5^{\prime \prime}$ seeing. These spectra were reduced and calibrated employing standard techniques in IRAF with standard stars from Strom (1977).

\section{RESULTS AND DISCUSSION}

As shown in Figure 1, a faint $\mathrm{H} \alpha$ emission shell at the location of J222557 can be seen amidst considerable diffuse $\mathrm{H} \alpha$ emission and numerous dust lanes. In Figure 2 we show enlargements of the $\mathrm{H} \alpha, \mathrm{H} \alpha-$ continuum, and [O III] $\lambda 5007$ images of this optical shell. The [O III] and $\mathrm{H} \alpha-$ continuum images are shown smoothed by a 5 point Gaussian. The optical shell has angular dimensions of $84^{\prime \prime} \times 70^{\prime \prime}$. This size is consistent with dimensions of $86^{\prime \prime} \times 75^{\prime \prime}$ reported by Morris et al. (2006) based on the Spitzer MIPS $24 \mu \mathrm{m}$ image which is also shown, smoothed by a 3 point Gaussian. No emission from the shell was seen in the [S II] $\lambda \lambda 6716,6731$ image despite being of similar or longer exposure times to those of $\mathrm{H} \alpha$ and [O III].

In Figure 3, we present our low-dispersion optical spectra of J222557 taken with a N-S oriented slit positioned along the eastern portion of the ring (see Fig. 2, middle panel). Narrow, unresolved (FWHM $<6 \AA$ ) emission lines of $\mathrm{H} \alpha$ and [N II] $\lambda \lambda 6548,6583$ were detected from both southern and northern limbs. No high-velocity emission was detected from either the nebula's shell or from any possible interior emission not visible in our narrow passband images. The observed $\mathrm{H} \alpha$ flux from the brighter southern limb knot is approximately $2.4 \times 10^{-16}$ erg $\mathrm{s}^{-1} \mathrm{~cm}^{-2}$ with an uncertainty around $50 \%$ due to non-photometric conditions. The [N II] $\lambda 6583$ line was stronger than the $\mathrm{H} \alpha$ for both northern and southern portions of the nebula's shell, with I([N $\mathrm{II}] 6583+$ $6548) / \mathrm{I}(\mathrm{H} \alpha) \simeq 2.7$ for the bright southern knot.

No [S II] $\lambda \lambda 6716,6731$ emission was detected in our spectra from the nebula, consistent with the lack of any emission seen on the narrow passband [S II] image. There was also no [O III] line emission detected in the spectrum despite the detected [O III] $\lambda 5007$ emission from the shell in the $[\mathrm{O}$ III $]$ image. We attribute the absence of detected [O III] in the spectrum due to a combination of a I([O III] $\lambda 5007 / \mathrm{I}(\mathrm{H} \alpha) \lesssim 1$, considerable line of sight extinction as suggested by the numerous dust lanes in the region (see Fig. 1), and a lower sensitivity of our spectral setup at $4500-5500 \AA$ compared to the $6000-7500 \AA$ region.

As noted by Morris et al. (2006), there is no previously known optical, radio, or X-ray nebula at the location of J222557. The object is not a cataloged planetary nebula (Acker et al. 1992) or a known Galactic nebula (Neckel \& Vehrenberg 1987), there is no coincident ra- 

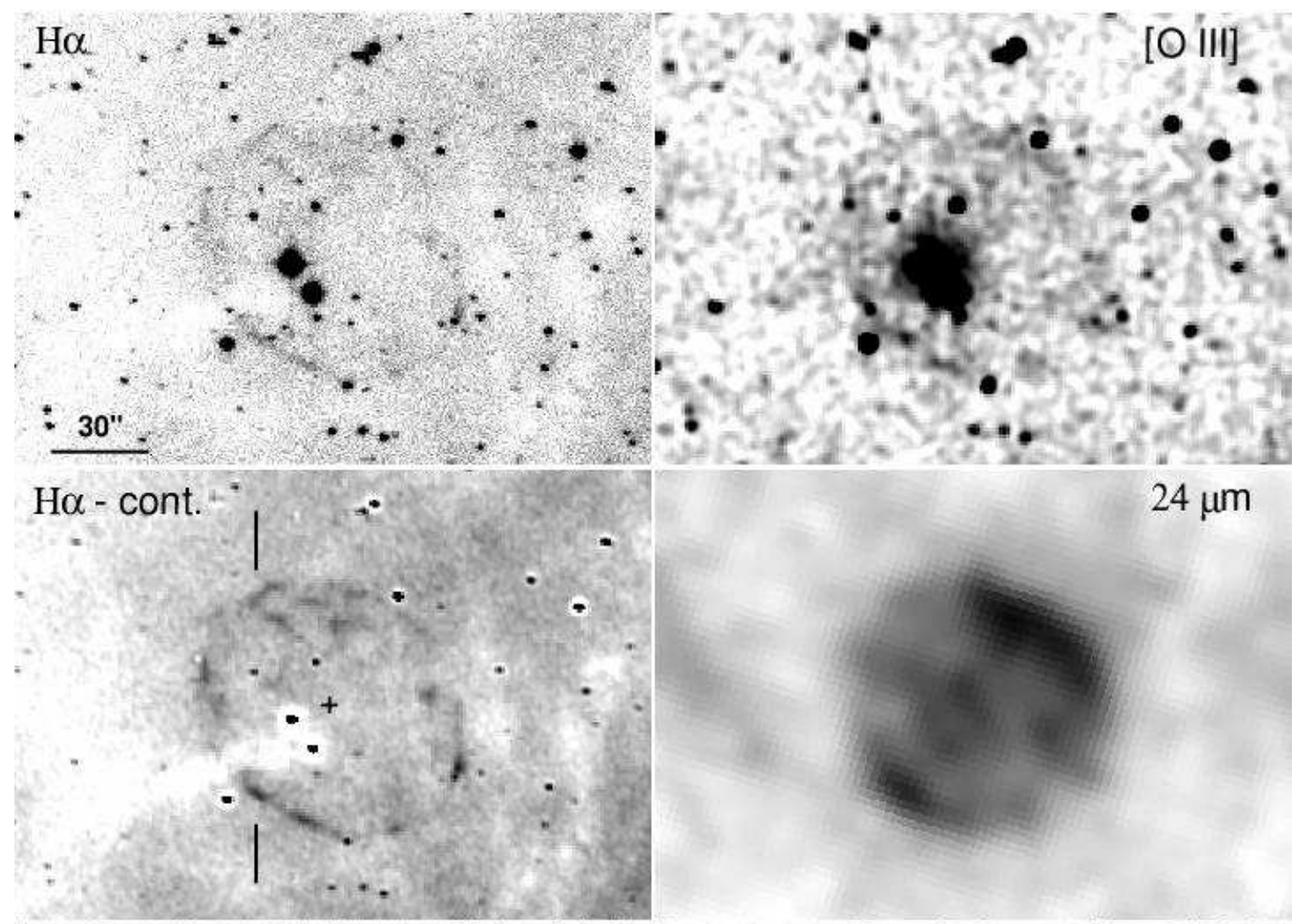

$24 \mu \mathrm{m}$

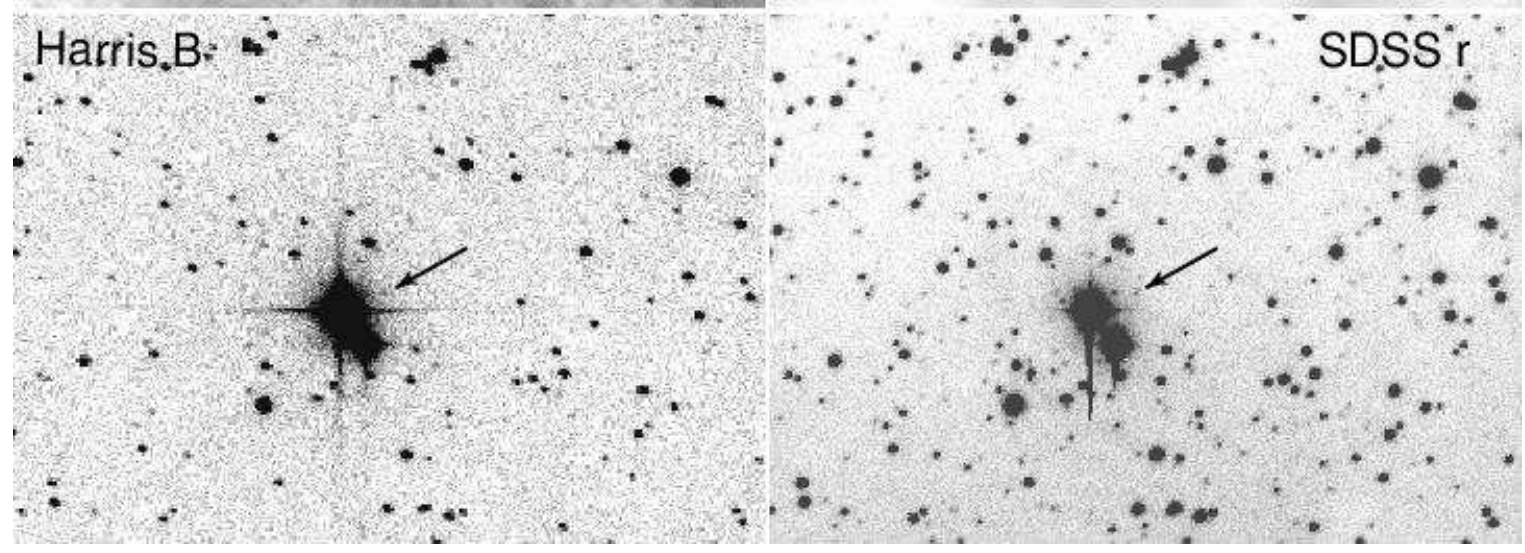

FIG. 2.- Enlarged optical and infrared images of J222557+601148. North is up and East to the left. Top row: MDM $1.3 \mathrm{~m}$ images showing faint $\mathrm{H} \alpha$ (left) and [O III] (right) emission shells. Middle row: $\mathrm{H} \alpha$ minus $6510 \AA$ continuum (left), and $24 \mu \mathrm{m}$ Spitzer MIPS (right). The position where we obtained the optical spectrum using a $\mathrm{N}-\mathrm{S}$ slit placed across the nebula is indicated in the H $\alpha$ minus 6510 $\AA$ continuum image. Bottom row: Broadband Harris B and SDSS $\mathrm{r}$ filter images with the central star PN (CSPN) candidate indicated by the arrows. This star's position relative to the emission shell is shown above in the H $\alpha$ minus continuum image by a ' + ' symbol.

dio source in either the Westerbork $325 \mathrm{MHz}$ Northern Sky Survey (WENSS) or the NRAO VLA Sky Survey 1.4 $\mathrm{GHz}$, and no associated X-ray emission is visible in the ROSAT All Sky Survey. The shell is also unlikely to be a Wolf-Rayet ring nebula due to the lack of a luminous point source in I band, 2MASS and IRAC images. Below, we discuss the nature of the J222557 nebula in light of these optical observations, first discussing a young supernova remnant scenario and then the object as a possible planetary nebula.

\subsection{A Young Supernova Remnant?}

Unusual infrared colors and a pure emission line $7.5-$ $37.5 \mu \mathrm{m}$ spectrum with no sign of dust continuum or hy- drogen emission lines led Morris et al. (2006) to examine the possibility of J222557 being a young supernova remnant (SNR). Although SNRs are usually relatively strong emitters of X-rays and nonthermal radio emissions, they argued that the object's lack of any such emissions did not exclude it from being identified as a SNR if the object were relatively distant and/or if the SNR were relatively young with little interaction with the ISM. They noted the nebula's size ( dia $\approx 80^{\prime \prime}$ ) was consistent with a young SNR at a distance $\sim 10 \mathrm{kpc}$ with an average expansion velocity of a few $1000 \mathrm{~km} \mathrm{~s}^{-1}$. Such a large distance would also place the object well above the Galactic plane ( $\mathrm{z} \simeq 400 \mathrm{pc}$ ) which would help explain a lack of ISM interaction and hence its low radio and X-ray luminosity. 


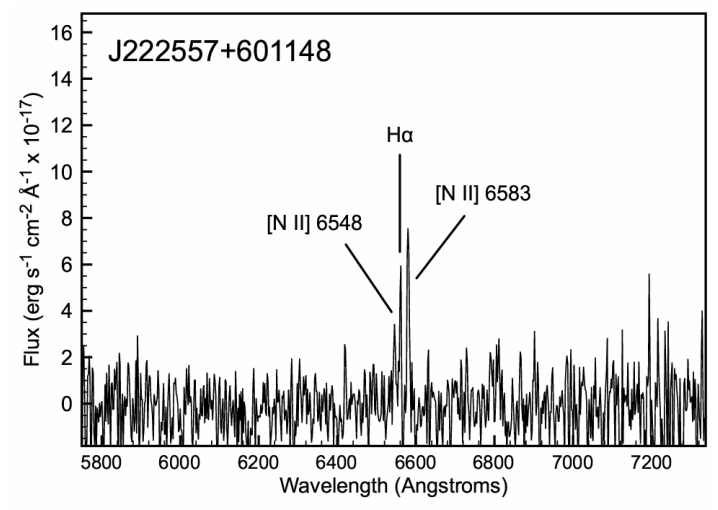

FIG. 3.- Optical spectrum of J222557+601148 showing narrow $\mathrm{H} \alpha$ and $[\mathrm{N}$ II] $\lambda \lambda 6548,6583$ line emissions.

As additional support for a SNR identification, Morris et al. (2006) cited J222557's similar morphology and size to the SMC remnant 1E $0102.2-7219$ and the young Galactic SNR Cassiopeia A (Cas A), both of which are oxygen-rich, core-collapse remnants. They noted 1E $0102.2-7219$ 's strong $24 \mu \mathrm{m}$ emission and absence in other Spitzer IR imaging bands (Stanimirović et al. 2005) much like that seen for J222557. Indeed, many SNRs exhibit strong $24 \mu \mathrm{m}$ emission and a faintness in shorter IR bands and this type of SED is illustrated in the 3.6, 8.0, and $24 \mu \mathrm{m}$ composite color image of the young galactic core-collapse SNR G11.3-0.3 shown in Carev et al. (2009).

Morris et al. (2006) also compared J222557's IRS spectrum with that of Cas A (Arendt et al. 1999). They found similar and consistent line ratios if J222557 had higher shock velocities than Cas A's $150-200 \mathrm{~km} \mathrm{~s}^{-1}$ in the ejected SN material of around $450-500 \mathrm{~km} \mathrm{~s}^{-1}$.

However, the presence of only narrow $\mathrm{H} \alpha$ and [N $\mathrm{II}]$ emissions with no high-velocity $\left(\mathrm{v} \geq 300 \mathrm{~km} \mathrm{~s}^{-1}\right)$ emission lines seen in either the optical or IR, plus no coincident X-ray or nonthermal radio emissions make a SNR interpretation unlikely. While much of the observed emission structure seen in our optical images is concentrated in a thin shell where large radial velocities would not be expected, there is some interior optical emission (Fig. 1) and one of the two Spitzer IRS spectra obtained by Morris et al. (2006) was taken of the shell's center and yet showed no hint of high-velocity.

Our optical spectrum also revealed no emission from [O I] $\lambda \lambda 6300,6364$ or [O II] $\lambda \lambda 7319,7330$ which would be expected if the nebula contained O-rich ejecta as seen in $1 \mathrm{E} 0102.2-7219$ and parts of Cas A. Moreover, the absence [S II] $\lambda \lambda 6716,6731$, which is the dominant line emission in much of Cas A's ejecta, also greatly weakens an analogy with this remnant.

Morris et al. (2006) also discussed the possibility that J222557 might be a young SNR associated with a thermonuclear Type Ia SN explosion, noting that strong 24 $\mu \mathrm{m}$ emission but weaker emission at $3.6-8 \mu \mathrm{m}$ has been observed in SNRs associated with this type of SN. For example, Borkowski et al. (2006) studied four LMC Type Ia remnants and found none were detected in the the Spitzer IRAC bands. Williams et al. (2006) found a similar situation in some outer regions of the LMC remnants N49 and N63A which were attributed to line emissions from [O VI] $25.9 \mu \mathrm{m}$ and [Fe II] 24.5 and $26.0 \mu \mathrm{m}$.

The morphology of the J222557 nebula certainly resembles the faint thin shells of young Type Ia SNRs such as Tycho and several young LMC remnants, and Morris et al. (2006) made a cautious comparison of J222557 to the bilaterally symmetric shell of the Type Ia SN 1006 remnant. Young Type Ia SNRs exhibit a spectrum dominated by low-velocity Balmer lines of hydrogen as a result of high-velocity shocks moving through a partially neutral medium leading to the production of strong narrow and much weaker broad Balmer lines before complete ionization occurs (Chevalier \& Raymond 1978; Chevalier et al. 1980; Heng 2010).

However, a young Type Ia SNR scenario for J222557 is also inconsistent with its observed optical spectrum. Elements other than hydrogen are also collisionally ionized in the postshock region and may emit line photons, but in the case of neutral atoms and relatively low-ionization ions, a line's luminosity is proportional to its ionization time and elemental abundance. This leads to relatively weak metal lines compared to the hydrogen Balmer lines. Thus the [N II] $\lambda 6583$ line emissions seen in the J222557 spectrum at levels actually stronger than $\mathrm{H} \alpha$ for both northern and south rims effectively rules out the optical nebula being due to a fast shock like that seen in the spectra of young Type Ia SNRs.

Finally, the lack of detected [S II] $\lambda \lambda 6716,6731$ in either our deep [S II] image or optical spectra of J222557 is also inconsistent with the J222557 nebula being an older, more evolved SNR. Due to an extended postshock cooling zone in remnants with shock velocities below 1000 $\mathrm{km} \mathrm{s}^{-1}$, the presence of strong [S II] emission is one of the chief identifiers of shock heated gas and has been widely used to discriminate between photoionized nebulae such as H II regions and planetary nebulae. Virtually all evolved SNRs show [S II]:H $\alpha \geq 0.4$ whereas H II regions typically exhibit values $\leq 0.2$ (Blair et al. 1981; Fesen et al. 1985).

\subsection{A Planetary Nebula?}

The axial symmetry and limb brightened emission observed in J222557 are strongly suggestive of a PN nature as Morris et al. (2006) themselves discuss. However, they rejected this identification based on its peculiar infrared colors, the absence of an IR bright central source often seen in PNe, and its Spitzer IRS spectrum which showed no continuum emission or hydrogen lines and no dust emission features commonly seen in PNe.

Nonetheless, a PN nature seems more likely given its optical properties. Emission line expansion velocities under $100 \mathrm{~km} \mathrm{~s}^{-1}$, relatively weak [S II] $\lambda \lambda 6716,6731$ emission $(\mathrm{I}([\mathrm{S} \mathrm{II}]) / \mathrm{I}(\mathrm{H} \alpha)<0.2$, ) and a morphology consistent with a limb brightened shell with some interior emission knots are properties frequently seen in PNe. The observed high $\mathrm{I}([\mathrm{N}$ II $] 6583+6548) / \mathrm{I}(\mathrm{H} \alpha)=2.7$ ratio is also often observed in $\mathrm{PNe}$ due to an overabundance of nitrogen in post-MS mass loss material (Acker et al. 1989, 1991).

Small $\left(<1^{\prime}\right)$ ring and shell morphology nebulae bright at $24 \mu \mathrm{m}$ but faint at $3.6-8.0 \mu \mathrm{m}$ are not unusual in the Galactic plane and some of these are suspected $\mathrm{PNe}$ (Carev et al. 2009; Flagev et al. 2009). J222557's observed infrared flux of less than $0.1 \mathrm{MJy} \mathrm{sr}^{-1}$ at the four IRAC channels $(3.6,4.5,5.8$, and $8.0 \mu \mathrm{m})$ but about 10 
MJy sr ${ }^{-1}$ at $24 \mu \mathrm{m}$ indicates a steeply rising SED. This SED is commonly seen for PNe due in part to significant continuum emission from warm dust (Zhang \& Kwok 2009). The bright appearance of PNe in MIPS $24 \mu \mathrm{m}$ images is due to both dust emission and [Ne V] $24.3 \mu \mathrm{m}$ and [O IV] $25.9 \mu \mathrm{m}$ line emissions (Zhang \& Kwok 2009).

The chief difficulty in assigning a PN identification to the J222557 nebula lies in an absence of appreciable dust continuum emission. The nebula's non-detection in the four IRAC $3.6-8.0 \mu \mathrm{m}$ images and the 70 or $160 \mu \mathrm{m}$ MIPS images (Morris et al. 2006) sets strong limits on the presence of warm or cool dust. The IRS spectrum of J222557 is consistent with the IRAC and MIPS images, showing a purely emission line spectrum with virtually no continuum or dust features, but with strong [O IV] 25.9 $\mu \mathrm{m}$ line emission explaining its detection in the MIPS 24 $\mu \mathrm{m}$ image.

However, Chu et al. (2009) found that the relative importance of nebular line emissions and dust continuum emission in the $24 \mu \mathrm{m}$ band for PNe depends on the temperature of the central star and distribution of dust and gas in the nebula. Using a sample of $28 \mathrm{PNe}$, they also found that smaller PNe exhibited $24 \mu \mathrm{m}$ emission that was more extended than their $\mathrm{H} \alpha$ emission and concluded that this extended $24 \mu \mathrm{m}$ emission was dominated by dust emission. Larger PNe, in contrast, show much weaker dust emission with the [O IV] line tending to dominate the emission in the MIPS $24 \mu \mathrm{m}$ band. These results are in accord with Stanghellini et al. (2007) who found the largest PN in the LMC and SMC exhibited the least dust continuum emission.

Our optical spectral data are also consistent with J222557 being a PN in terms of its size. We measured $\mathrm{v}_{\mathrm{LSR}}=-70 \pm 20 \mathrm{~km} \mathrm{~s}^{-1}$ from the observed $\mathrm{H} \alpha$ line emission, which is consistent with galactic rotation at its $l=105.8$ and a location inside the Perseus Arm at a distance of $2-3 \mathrm{kpc}$ (Russeil et al. 2007). With a $b=2.3$, a distance of $\approx 2.5 \mathrm{kpc}, \mathrm{J} 222557$ would lie some $100 \mathrm{pc}$ above the Galactic plane. At a distance of 2 to $3 \mathrm{kpc}$, J222557's angular radius of 40" implies a linear radius of around $0.5 \mathrm{pc} \times(\mathrm{d} / 2.5 \mathrm{kpc})$ which is near the median size of PNe (Cahn et al. 1992; van de Steene \& Zijlstra 1994; Ciardullo et al. 1999; Bensby \& Lundström 2001; Frew \& Parker 2010). Interestingly, some $20 \%$ of all PNe are not detected in the radio and a size $\geq 0.5 \mathrm{pc}$ would indicate a well evolved PN which might help explain J222557's lack of detected radio emission.

Finally, we have identified a possible planetary nebula central star (CSPN) candidate. A blueish point source near J222557's center is detected in our B, $g^{\prime}$ and $r^{\prime}$ images, with $m_{g^{\prime}}=22.8 \pm 0.2$ and $m_{r^{\prime}}=22.4 \pm 0.2$. Figure 2 (bottom row) shows our Harris B and SDSS $r^{\prime}$ filter images of the nebula with the candidate central exciting star indicated. This star's location places it nearly centered in the nebula as shown by the cross in the $\mathrm{H} \alpha-$ continuum difference image.

With an uncertain distance and line of sight extinction, and only very weak detections in our broadband images, it is difficult to accurately assess the CSPN candidate's intrinsic color or luminosity. At $m_{V} \approx 22.5$, it would rank among the faintest Galactic CSPN known (Tylenda et al. 1991). However, adopting a distance of $2.5 \mathrm{kpc}$ and an $\mathrm{A}_{\mathrm{V}}=4 \mathrm{mag}$ based $\mathrm{H}$ I measurements for $l=105^{\circ} .8$ and $b=2.3$ and conversions of $\mathrm{N}(\mathrm{H})$ into $\mathrm{A}_{\mathrm{V}}$ values for a typical gas to dust ratio (Bohlin et al. 1978; Predehl \& Schmitt 1995), the candidate would have $\mathrm{M}_{\mathrm{V}} \simeq 6.5$, a value quite consistent for CSPN in evolved PNe (Phillips 2005; Benedict et al. 2009). Moreover, given the strength of [O IV], the nebula is likely to have considerable He II emission which would place a lower limit of $\simeq 60,000 \mathrm{~K}$ for the star's effective temperature (Kaler \& Jacoby 1989).

\section{CONCLUSIONS}

Morris et al. (2006) tentatively identified the Galactic nebula J222557+601148 as a young supernova remnant (SNR) based Spitzer Galactic First Look Survey images. However, $\mathrm{H} \alpha$, [O III], [S II] images and low dispersion optical spectra reveal it to only exhibit narrow $\mathrm{H} \alpha$ and [N II] $\lambda \lambda 6548,6583$ line emissions with no evidence of broad or high-velocity line emissions.

The absence of any high-velocity optical or infrared features, the presence of relatively strong [N $\mathrm{II}]$ emissions, a lack of detected [S II] emission which would indicate the presence of shock-heated gas, plus no coincident X-ray or nonthermal radio emissions suggest the nebula is unlikely to be a SNR, young or old. Instead, it is likely a faint, high-excitation $\mathrm{PN}$ as its elliptical morphology would suggest, lying at a distance $\sim 2.5 \mathrm{kpc}$ with strong [O IV] emission rather than dust continuum emission dominating it detection in $24 \mu \mathrm{m}$ Spitzer images. We have identified a possible central star candidate with $m_{r^{\prime}} \simeq 22.4$.

We like to thank D. Green for encouraging us to write this work up, and the anonymous referee for helpful comments which improved the manuscript.

\section{REFERENCES}

Acker, A., Jasniewicz, G., Koeppen, J., \& Stenholm, B. 1989, A\&AS, 80, 201

Acker, A., Raytchev, B., Koeppen, J., \& Stenholm, B. 1991, A\&AS, 89, 237

Acker, A., Marcout, J., Ochsenbein, F., Stenholm, B., \& Tylenda, R. 1992, Garching: European Southern Observatory, 1992,

Arendt, R. G., Dwek, E., \& Moseley, S. H. 1999, ApJ, 521, 234

Benedict, G. F., et al. 2009, AJ, 138, 1969

Bensby, T., \& Lundström, I. 2001, A\&A, 374, 599

Blair, W. P., Kirshner, R. P., \& Chevalier, R. A. 1981, ApJ, 247, 879

Bohlin, R. C., Savage, B. D., \& Drake, J. F. 1978, ApJ, 224, 132

Borkowski, K. J., et al. 2006, ApJ, 642, L141

Cahn, J. H., Kaler, J. B., \& Stanghellini, L. 1992, A\&AS, 94, 399
Carey, S. J., et al. 2009, PASP, 121, 76

Chevalier, R. A., \& Raymond, J. C. 1978, ApJ, 225, L27

Chevalier, R. A., Kirshner, R. P., \& Raymond, J. C. 1980, ApJ, 235,186

Chu, Y.-H., et al. 2009, AJ, 138, 691

Ciardullo, R., Bond, H. E., Sipior, M. S., Fullton, L. K., Zhang, C.-Y., \& Schaefer, K. G. 1999, AJ, 118, 488

Flagey, N., Billot, N., Carey, S., Noriega-Crespo, A., Shenoy, S., Mizuno, D., Paladini, R., \& Kraemer, K. 2009, BAAS, 41, 762 Fesen, R. A., Blair, W. P., \& Kirshner, R. P. 1985, ApJ, 292, 29 Fesen, R. A., et al. 2006, ApJ, 645, 283

Frew, D. J., \& Parker, Q. A. 2010, Pub. Astr. Soc. Australia, in press (arXiv:1002.1525) 
Green, D. A., Reynolds, S. P., Borkowski, K. J., Hwang, U., Harrus, I., \& Petre, R. 2008, MNRAS, 387, L54

Green, D. A. 2009, Bulletin of the Astronomical Society of India, 37,45

Heng, K. 2010, Pub. Astr. Soc. Australia, 27, 23

Kalberla, P. M. W., Burton, W. B., Hartmann, D., Arnal, E. M., Bajaja, E., Morras, R., Poumlppel, W. G. L. 2005, A\&A, 440, 775

Kaler, J. B., \& Jacoby, G. H. 1989, ApJ, 345, 871

Morris, P. W., Stolovy, S., Wachter, S., Noriega-Crespo, A., Pannuti, T. G., \& Hoard, D. W. 2006, ApJ, 640, L179

Morgan, C. W., et al. 2005, AJ, 129, 2504

Neckel, Th., \& Vehrenberg, H. 1987 "Atlas of Galactic Nebulae" (Duesseldorf: Treugesell-Verlag), Vol. 2

Phillips, J. P. 2005, MNRAS, 357, 619

Predehl, P., \& Schmitt, J. H. M. M. 1995, A\&A, 293, 889

Reynolds, S. P., Borkowski, K. J., Green, D. A., Hwang, U., Harrus, I., \& Petre, R. 2008, ApJ, 680, L41

Russeil, D., Adami, C., \& Georgelin, Y. M. 2007, A\&A, 470, 161
Smith, J. A. 2002, AJ, 123, 2121

Stanghellini, L., García-Lario, P., García-Hernández, D. A., Perea-Calderón, J. V., Davies, J. E., Manchado, A., Villaver, E., \& Shaw, R. A. 2007, ApJ, 671, 1669

Stanimirović, S., Bolatto, A. D., Sandstrom, K., Leroy, A. K., Simon, J. D., Gaensler, B. M., Shah, R. Y., \& Jackson, J. M. 2005, ApJ, 632, L103

Strom, K. M. 1977, Kitt Peak National Observatory

Memorandum, Standard Stars for Intensified Image Dissector Scanner Observations (Tucson: KPNO)

Thorstensen, J. R., Fesen, R. A., \& van den Bergh, S. 2001, AJ, 122,297

Tylenda, R., Acker, A., Raytchev, B., Stenholm, B., \& Gleizes, F. 1991, A\&AS, 89, 77

van de Steene, G. C., \& Zijlstra, A. A. 1994, A\&AS, 108, 485

Williams, R. M., Chu, Y.-H., \& Gruendl, R. 2006, AJ, 132, 1877

Zhang, Y., \& Kwok, S. 2009, ApJ, 706, 252 